\title{
Closing the Gap Between Theory and Observations of Venus Atmospheric Dynamics with New Measurements
}

\author{
Amanda S. Brecht \\ NASA Ames Research Center \\ (650) 604-2983 \\ Amanda.s.brecht@nasa.gov
}

Stephen H. Brecht ${ }^{1}$, Janet Luhmann ${ }^{2}$, Josette Bellan ${ }^{3}$, Kandis-Lea Jessup ${ }^{4}$, Thomas Navarro ${ }^{5,6}$, Sébastien Lebonnois $^{7}$, Stephen W. Bougher ${ }^{8}$, Yingjuan $\mathrm{Ma}^{5}$, Helen F. Parish ${ }^{5}$

${ }^{1}$ Bay Area Research Corp.

${ }^{2}$ UC Berkeley

${ }^{3}$ Jet Propulsion Laboratory

${ }^{4}$ Southwest Research Institute
${ }^{5}$ UCLA
${ }^{6}$ McGill Univ.
${ }^{7}$ Laboratoire de Météorologie Dynamique/IPSL
${ }^{8} \mathrm{U}$. of Michigan

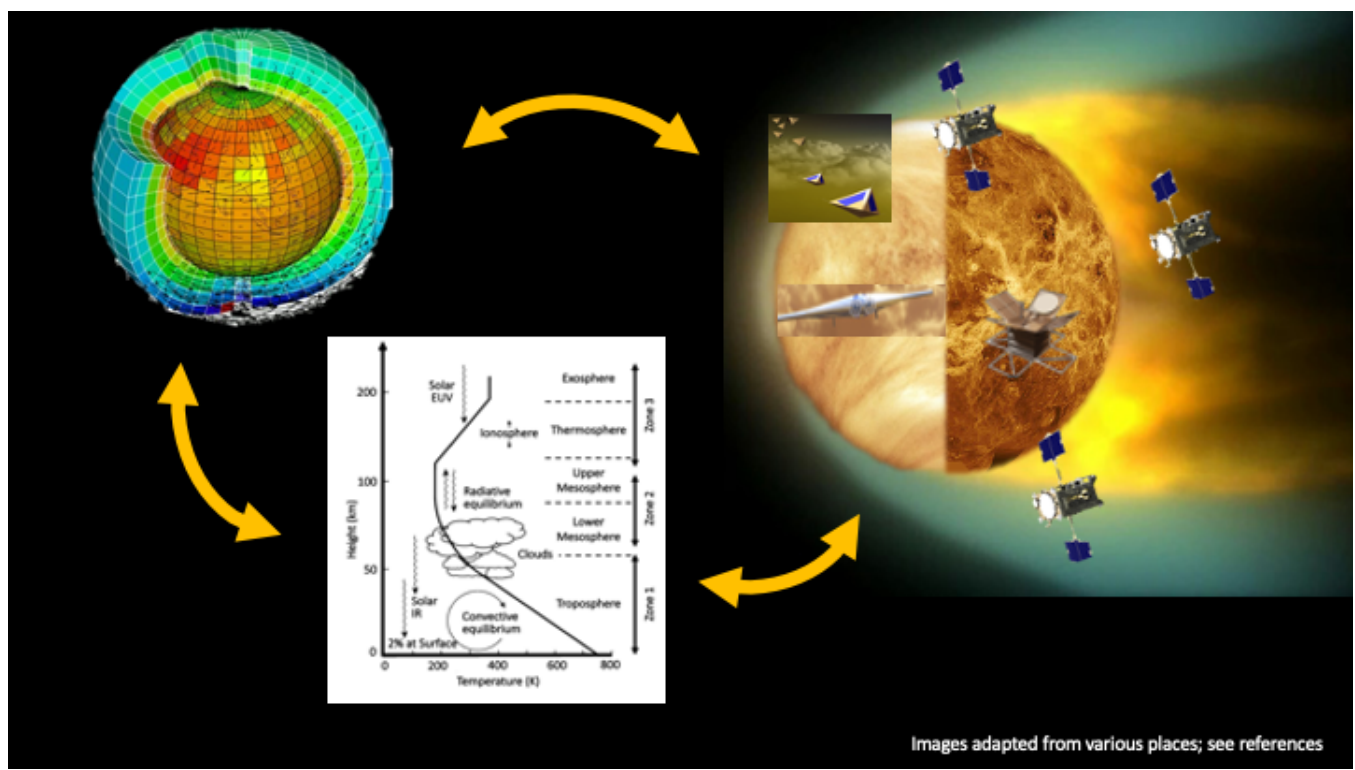

Name (Institution)
Hiroki Ando (Kyoto Sangyo Univ., Japan)
Kevin Baines (JPL)
Pat Beauchamp (JPL)
Mark Bullock (Southwest Research Institute)
Glyn Collinson (NASA GSFC/ Catholic Univ. of America)
David Crisp (JPL)
Shannon Curry (UC Berkeley)
Chuanfei Dong (Princeton)
Itziar Garate-Lopez (Universidad del País Vasco / Euskal
Herriko Unibertsitatea, Bilbao, Spain)
Gabriella Gilli (Instituto de Astrofísica e Ciências do
Espaço (IA), Lisbon, Portugal)
Martha Gilmore (Wesleyan University)
Dmitry Gorinov (Space Research Institute (IKI), Russia)
Candace Gray (NM State Univ. /Apache Point Observatory)
Thomas Greathouse (SWRI)
Scott Guzewich (NASA GSFC)
Devanshu Jha (MVJ College of Engineering)

\section{Name (Institution)}

Stephen Kane (UC Riverside)

Siddharth Krishnamoorthy (JPL)

Stephen Ledvina (UC Berkeley)

Stephen Lewis (The Open University, UK)

Liming Li (Univ. of Houston)

Robert Lillis (UC Berkeley)

Ryan McCabe (Hampton Univ.)

Kevin McGouldrick (Univ. of CO., Boulder)

Frank Mills (SSI)

Christopher Parkinson (SSI)

Jason Rabinovitch (JPL)

Emilie Royer (PSI)

Norihiko Sugimoto (Keio Univ., Japan)

Melissa Trainer (NASA/GSFC)

Michael Way (NASA GISS)

Shaosui Xu (UC Berkeley)

Masaru Yamamoto (Kyushu Univ., Japan) 


\section{Executive Summary:}

The purpose of this white paper is to advocate for a new paradigm in Venus atmospheric missions producing new state-of-the-art atmospheric measurements. It is our closest neighbor in size and distance and yet has an atmosphere that is a caustic mixture of sulfur-laden gases whose opacity, surface pressure, and temperature have defied its close exploration. It also has a surface that is equally hostile and has both affected, and been affected by the atmosphere over time. Furthermore, the atmosphere has a zonal flow pattern that rotates faster than the solid body rotation. The relative speed of the zonal flows varies with altitude in a manner that seems to be organized into 3 different (yet possibly interacting/linked) zones of flow, for reasons not fully understood (see Fig. 1, Section 1 below, e.g. Sánchez-Lavega et al. 2017 and Taylor et al. 2018).

Full understanding of the mechanisms that support and maintain the atmospheric superrotation, altitude flow pattern zones and other key aspects of Venus' atmospheric dynamics remains among the most intriguing puzzles of the solar system. In spite of past missions and other observations, complementary data analyses and numerical modeling of various components of Venus' atmospheric dynamics, a complete understanding of Venus' atmosphere as a coupled radiative-dynamics system remains elusive.

The Venus community is applying the latest technologies and numerical tools to the exploration of Venus' atmosphere. As a result of this effort, a new set of questions needs to be addressed in order to advance the physical understanding of the Venusian atmosphere. For example:

- How are the different zones of Venus' circulation patterns related?

- How does the solar environment impact the zonal flow of Venus' upper atmosphere?

- How is Venus' nightside ionosphere maintained?

- What are the characteristics of Venus' cloud and haze layers?

- What can wave patterns and propagation teach us about the relative importance of stability regions in Venus' lower atmosphere?

- What impact does the wave propagation have on Venus' circulation patterns as a function of altitude (zone)?

All open atmospheric questions at Venus are dependent on our ability to assess coupling between external drivers and internal processes on Venus' atmospheric state. Numerical tools that accurately assess this coupling on Venus' atmospheric state are essential for resolving the open Venus science questions. Currently, there is a suite of physics-based time dependent threedimensional (3D) numerical tools tailored for Venus' atmosphere that are capable of simulating a multitude of atmospheric properties. These numerical tools/simulations are highlighting regions where the current understanding of nonlinear interactions fail and need to be guided and constrained with new "modern" observations.

A new era of simultaneous and synergistic observation strategies ("modern" observations) are needed to explore links between day and nightside processes and processes extending over broad vertical (altitude) regions (e.g. Tamppari et al. and McGouldrick et al. white papers). The extended vertical observations must address spatial regions that intersect with areas of variable wind speed, circulation pattern, chemical composition, aerosol density and/or thermal structure.

New missions to Venus must include the state-of-the-art atmospheric measurements needed to address the gaps in knowledge illuminated by current numerical simulations. Addressing these gaps in knowledge will further provoke follow-on scientific questions that will advance, shape and refine our understanding of Venus' global atmospheric properties. 


\section{Scientific Issues and Challenges:}

Traditionally, scientists (whether conducting observations, experiments, or developing new theoretical tools) view an atmosphere to consist of delimited and defined vertical regions. Planetary atmospheres are divided into troposphere, stratosphere, mesosphere, thermosphere, and exosphere as derived from Earth's thermal structure. Figure 1 illustrates these regions with respect to Venus' mean thermal structure. For Venus, there is a lack of understanding on how these atmospheric regions interact. In addition, there is a growing disagreement between past observations and sophisticated numerical simulations. This indicates a lack of understanding regarding the interaction between or among vertical regions.

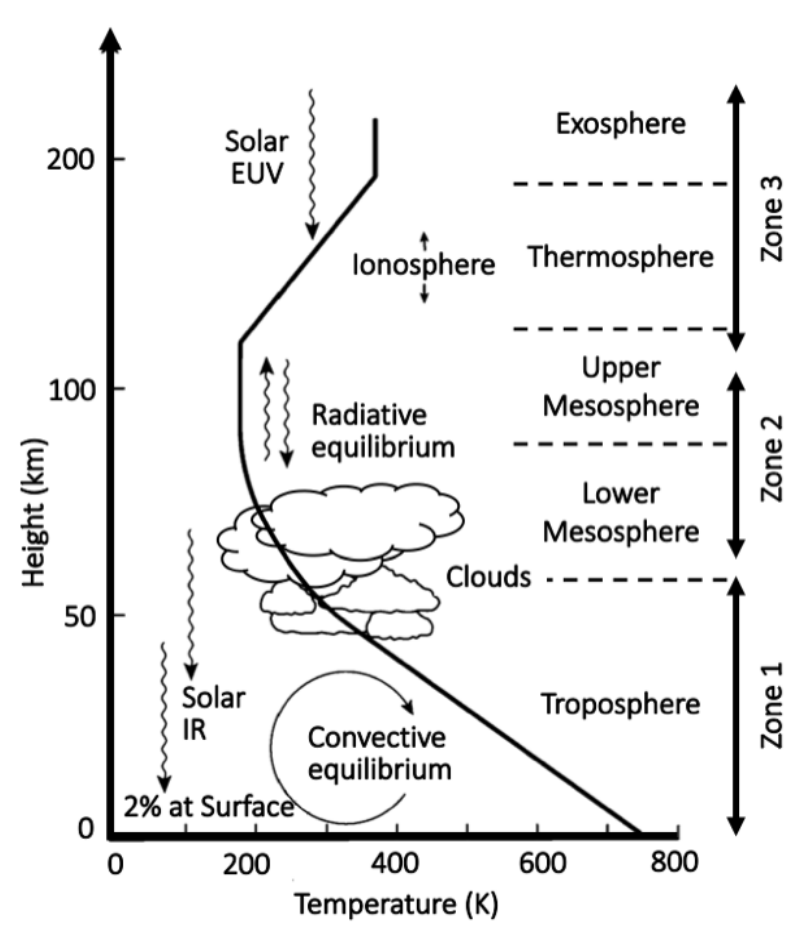

Figure 1: Average thermal profile of Venus' atmosphere with labels of the estimated locations of energy transport processes (adapted from (Taylor et al., 2018)).

One of the longest standing unexplained facts regarding Venus, is the observed zonal super-rotation of the atmosphere (superrotation is defined as occurring when the zonal atmospheric flow is faster than the planets solid body rotation) (cf. Schubert et al., 2007). The equatorial cloud tops circle the planet in 4 Earth days compared to Venus' solid body rotation rate of 243 Earth days. The current understanding of the zonal super-rotating atmosphere is mainly derived from the, Soviet Union Venera and VEGA missions, NASA Pioneer Venus mission (PVO), and the ESA Venus Express mission (VEX) with augmentations from the current JAXA Akatsuki mission (cf. Gérard et al., 2017; Horinouchi et al., 2020; Imamura et al., 2020; Morellina et al., 2020; Read \& Lebonnois, 2018; Sánchez-Lavega et al., 2017). For simplicity of explanation within this white paper, the zonal atmospheric flow is broken up into 3 zones based upon observations and shown in Figure1. Zone 1 is from the surface to $\sim 70 \mathrm{~km}$ and is dominated by flow in the direction of the Venus' rotation. The zonal flow velocity is at most a few $\mathrm{m} / \mathrm{s}$ near the surface and increases to $\sim 100 \mathrm{~m} / \mathrm{s}$ at the cloud tops (i.e. super-rotation motion). Zone 2 is from $\sim 70 \mathrm{~km}$ to $\sim 120 \mathrm{~km}$ and the dominant zonal flow changes and is highly variable. The zonal flow transitions from a dominant super-rotation motion to a dominant day-to-night motion. Above $\sim 70 \mathrm{~km}$ the super-rotation is reduced while the pressure gradient driven day-to-night zonal flow begins to dominate. A second transition occurs near the top of Zone 2 and into Zone 3 ( $\sim 120 \mathrm{~km}$ and above). Here the zonal flow transitions to a varying combination of day-to-night motion and super-rotation motion. The nature of these zones is part of the long-standing unexplained facts about Venus' atmosphere. Are the super-rotating regions above and below the clouds connected?

From Venus mission observations, scientists are beginning to unravel the mystery drivers of Venus' zonal super-rotation. An example of newly found insights comes from observational evidence of thermal perturbations at the cloud tops. It was a common assumption that waves from the Venusian surface do not reach above the cloud tops, thus the lower atmosphere was not strongly 
connected to the upper atmosphere via waves. However, the JAXA Akatsuki mission observed a thermal brightness perturbation, which corresponds to specific topographic features. Additionally, numerical simulations have now demonstrated topographic driven waves do reach the cloud tops after incorporating sub grid-scale parameterization of the topography (Fukuhara et al., 2017; Kouyama et al., 2017; Navarro et al., 2018). How high in altitude do these waves propagate? What does this mean for the stability of the lower atmosphere?

Adding another piece to the mystery, Venus Express observed an asymmetric solar wind and ionospheric ion flow near Venus (Lundin et al., 2011). This is proposed to be due to Venus' orbital motion transverse to the solar wind with feedbacks from the neutral atmosphere through ionneutral drag. This theory has not been fully numerically tested, yet. In fact, a provocative question comes from the realization that the orbital velocity of Venus is roughly $10 \%$ of the nominal solar wind velocity and does not have a magnetic field. Does this additional velocity component change how Venus' atmosphere interacts with the solar wind and does such a change in interaction lead to some of the observations made in the neutral atmosphere circulation patterns?

The waves driven by topography and the solar wind represent external drivers (boundary conditions) on Venus' atmosphere and impact the zonal flow. A bigger question is how do these drivers interact with each other and with other internal processes such as energy deposition and damping (i.e. cloud/haze formation, atmospheric waves, etc.)?

\section{Drivers for New Mission Observations (Needed Observations):}

The above-stated questions, and many additional ones (as formulated by VEXAG19), indicate that additional (preferably simultaneous) observations of multiple parameters (such as temperature, density, composition, motion, and solar input) in multiple dimensions (vertical, horizontal, and temporal) are necessary. Observations are especially needed to explore the connections across vertical regions where directionality in the zonal flow pattern(s) and/or the slope of temperature gradient become convergent or divergent. Simultaneous observations of different parameters, as well as simultaneous observations of the same parameter at different locations, etc. are required to support the level of numerical model and simulation complexity required to assess/investigate the mechanics of Venus' dynamics. For example, the coupling of different altitude/local time regions seems to be one of the sources of disagreement between past data and current simulations. This suggests that observations of the lower atmosphere, the upper atmosphere, and the ionosphere must occur simultaneously. This requires that future Venus missions produce data more connected in space and time than past missions. "Modern" Venus observations must employ a new/more advanced mission architecture paradigm/payload producing observations that can unambiguously characterize the relative impact and coupling of the external and internal physical forces that control the physical nature of Venus' dynamics relative to local time, altitude and latitude.

Currently, several Venus time dependent 3D numerical tools (producing numerical simulations) are being utilized to investigate the physical processes controlling Venus' atmosphere and to interpret past observational profiles and localized data. Some of these numerical tools are shown in Table 1 and it is clear most numerical tools are specific to a stratified vertical region. Reasons for these stratifications include: (i) the governing equations and associated simplifying assumptions are only valid in specific vertical regions of the atmosphere; (ii) limited computational resources (insufficient memory or speed); (iii) intentional spatial constraint to match observations from limited (localized) areas; and lastly (iv) scientific interest in a specified altitude region. As numerical tools' capabilities are progressing, the simulations are exhibiting sensitivities to 
boundaries, resolution, and additional physics not initially included in the governing equations of the simulations. These sensitivities need to be understood and constrained. The simulations need coupled/synergistic observations to advance the understanding of the Venus atmosphere. Here are examples of observations needed based upon the sensitivities of the simulations:

\section{External Drivers/Boundary Conditions}

- Solar Input (energy deposition from infrared, solar UV and solar EUV fluxes): The main energy driver of most atmospheres is the Sun. However, the Sun is variable and how the atmosphere absorbs and distributes the energy is sensitive to time and location. For instance, there is little information in regards to the net solar energy deposition below the clouds. Atmospheric dynamics are tightly coupled to radiative effects. Thus, it is exceedingly important to make these measurements at Venus instead of extrapolating from Earth or other locations.

- Near Surface Properties (temperature, pressure, winds, and composition): There are very few direct observations of the basic atmospheric properties below $\sim 10 \mathrm{~km}$ due to the extreme Venus environment. These properties are important to understand the interaction between the surface and atmosphere. Moreover, information about these properties leads to knowledge about the heat and angular momentum exchange that drives temperature and wind structure in the atmosphere. New technologies have been developed and are capable of surviving Venus' harsh environment to make these measurements (VEXAG19_Tech).

\section{$\underline{\text { Internal Processes }}$}

- Temperature and Wind: These basic atmospheric properties are intimately connected to the dynamics, such that temperature gradients drive dynamics and 3D winds are tracers of dynamics. Dynamical observations of temperature and wind, covering different regions simultaneously are extremely important.

- Cloud and Haze Layers: Full hemispheric observations are needed for the cloud layers with continuous monitoring to provide information on the mean wind field, thermal tides, planetaryscale waves, and small-scale gravity waves. Simulations that reproduce the super-rotating lower atmosphere are found to be very sensitive to the mean meridional circulation and planetary-scale waves, that feed into the middle atmosphere. Observations are needed to help understand the sensitivity to these couplings.

- Cloud Particle Properties and Spatial Distribution: Simulations have shown small changes in particle size and spatial distribution will greatly affect the transfer of radiation and thus the dynamics in the middle atmosphere.

- Chemical Species Distributions (e.g. $\mathrm{CO}_{2}, \mathbf{S O x}, \mathbf{C O}, \mathbf{O}$ ): These observations can ascertain radiative forcing sensitivity (e.g. UV absorber(s) and the controlling factors in the $\mathrm{CO}_{2} 15-\mu \mathrm{m}$ cooling). In addition, it can provide circulation information due to transport lifetimes being shorter than the certain species chemical lifetime (i.e. tracers of the circulation).

- Thermal and Non-Thermal emissions (i.e. dayglow, nightglow, aurora): Emissions are incredibly useful to help trace circulation patterns. Having continuous observations and/or simultaneous observations in 3D (spatial) of the emissions can provide information on variability of the emission (i.e. dynamics) but also wave information. See the Royer et al. white paper for more details.

- Ion/Electron Densities: Measurements are needed to help understand how the plasma and the neutral atmosphere are coupled. The measurements will provide information about the energy 
and momentum transfer from the solar wind into the upper atmosphere. Moreover, it will help to understand how the nightside ionosphere is maintained which is not known.

- Plasma Distribution Functions: This would contribute to the understanding of the energy inflow and outflow of Venus' atmosphere via energetic particles. These measurements will provide ion/electron temperatures, further illuminating the process that couples plasma and neutrals. From the ion/electron temperature one could investigate some of the drivers of the chemistry in the ionosphere.

- Magnetic Fields: Magnetic fields are the main conduit that connects the Interplanetary Magnetic Field (IMF) energy flow to the ionosphere; via currents that are induced in the ionosphere. They drive the currents and particle motions, which interact with the ionosphere and thus neutral atmosphere. This fundamental quantity needs to be measured if one hopes to understand the ionosphere of a planet.

This short list of needed observations will begin to close the gap between theory and observations and thus accelerate the scientific endeavor of understanding Venus' atmosphere.

\section{Connection between Observations and Numerical Simulations:}

The scientific endeavor is often thought of as a progressive process of observation followed by development of understanding and then using the recently obtained understanding to make predictions particularly in parametric regions where observations have not been previously made. Subsequent observations are then used to test the prediction and the basic understanding of the system. Thus, there is a cyclical dependence between observations and numerical simulations.

In the case of planetary atmosphere/ionosphere dynamics, the "understanding" portion of the scientific endeavor is often embodied in large complex numerical simulations that solve coupled non-linear differential equations within multi-dimensional numerical models. Where once they might be one dimensional time dependent simulations, they are now often time dependent 3D simulations and include not just dynamics driven by heat but chemistry and coupling to clouds and other states of matter (i.e. plasmas). These simulations are used as a framework for establishing a global context from spatially-limited and temporally-limited individual observations.

This cyclical scientific endeavor has been applied many times to Earth based atmospheric questions. Earth atmospheric science has demonstrated the success of utilizing "modern"/advanced observations in conjunction with numerical models to advance the state of the art in understanding the basic physics that controls Earth's weather and climate. The methodology is deemed successful enough that results derived from Earth atmosphere simulations (stratified according to specified science investigation goals) are used for pure/fundamental scientific advancement as well as to inform national public policy. The cyclical scientific endeavor needs to be strongly pursued for Venus. Similar advancements in Venus atmospheric science may be achieved provided future Venus missions are capable of acquiring the detailed observations (outlined in Section 2) which are essential for advancing our understanding of Venus' coupled radiative-dynamics processes.

\section{The Significance of Venus Research:}

The dynamics, momentum budget, and energy budget in a real atmosphere often do not follow the physical and numerical assumptions made within current simulations. Current numerical simulations indicate we do NOT understand how Venus' atmosphere works from the 
surface to space (see Fig. 1). While the available numerical simulation tools may be used to address the open puzzles in Venus atmospheric science, this work requires adequate observational data to assess how the physical constraints on the simulations correlate to real Venus atmosphere conditions. Such data-modeling interaction will also guide the simplifying assumptions of the simulations--i.e., to define the level of complexity of physics equations and the number of physical processes to include in the numerical model. In this modern age of computing (increased memory size and speed), the capability to produce full atmosphere simulations based on detailed physics equations and packages is beginning to be possible.

In the interest of comparative climatology, the Venusian atmospheric numerical tools are derived from Earth atmospheric numerical tools and therefore test the accuracy of the solution of the terms in the Earth based simulations. This is due to the importance of various driving terms changing between Earth and Venus (such as thermal transport and rotational forces). Continued comparisons between Earth and Venus simulations will be insightful for understanding both planetary atmospheres. Moreover, Earth (or equivalently, Venus) sized planets are key prototypes for targeted exoplanet studies. Therefore, a reliable base understanding of the Venusian physical constraints required to reasonably reproduce observational tracers of the dynamics and the processes controlling the dynamics will directly influence the development of $3 \mathrm{D}$ exoplanet atmospheric simulations.

Table 1: A list of the current time dependent three-dimensional models. [Rv = Venus Radius].

**References can be found at the end or in these overview chapters: Gérard et al., 2017; Ledvina et al., 2008; Sánchez-Lavega et al., 2017.

\begin{tabular}{|c|c|c|c|}
\hline Type/Name & Name / Affiliation & Altitude Range & Reference** \\
\hline General Circulation Model & WRF & $0-90 \mathrm{~km}$ & Richardson et al., 2007 \\
\hline General Circulation Model & $\begin{array}{l}\text { UK Meteorological } \\
\text { Office Unified } \\
\text { Model/ Oxford }\end{array}$ & $0-90 \mathrm{~km}$ & Lee et al., 2005; 2007 \\
\hline General Circulation Model & NCAR CAM & $0-95 \mathrm{~km}$ & Parish et al. 2011 \\
\hline General Circulation Model & CCSR/NIES & $0-90 \mathrm{~km}$ & $\begin{array}{l}\text { Yamamoto and } \\
\text { Takahashi, } 2003\end{array}$ \\
\hline General Circulation Model & FMS - Spectral & $0-90 \mathrm{~km}$ & Lee and Richardson, 2012 \\
\hline General Circulation Model & IPSL & $0-150 \mathrm{~km}$ & $\begin{array}{l}\text { Lebonnois et al., } 2010 \text {; } \\
\text { Gilli et al., } 2017\end{array}$ \\
\hline General Circulation Model & AFES Venus & $0-120 \mathrm{~km}$ & $\begin{array}{l}\text { Sugimoto et al, 2014; } \\
\text { Takagi et al., } 2018\end{array}$ \\
\hline $\begin{array}{l}\text { Thermospheric General } \\
\text { Circulation Model }\end{array}$ & $\begin{array}{l}\text { Tohoku University, } \\
\text { Japan }\end{array}$ & $80-180 \mathrm{~km}$ & Hoshino et al. 2012 \\
\hline $\begin{array}{l}\text { Thermospheric General } \\
\text { Circulation Model }\end{array}$ & $\begin{array}{l}\text { VTGCM / } \\
\text { Univ. of Michigan, } \\
\text { NASA ARC }\end{array}$ & $70-300 \mathrm{~km}$ & $\begin{array}{l}\text { Bougher et al., 1988; } \\
\text { Brecht et al., } 2011\end{array}$ \\
\hline $\begin{array}{l}\text { MHD - multispecies single- } \\
\text { fluid }\end{array}$ & BAT-S-RUS & $100 \mathrm{~km}-24 \mathrm{Rv}$ & Ma et al., 2013 \\
\hline Hybrid & HALFSHEL & $100 \mathrm{~km}-3 \mathrm{Rv}$ & $\begin{array}{l}\text { Brecht and Ferrante, 1991; } \\
\text { Ledvina et al., } 2016\end{array}$ \\
\hline Hybrid & HYB & $400 \mathrm{~km}-4 \mathrm{Rv}$ & $\begin{array}{l}\text { Kallio and Janhunen 2003; } \\
\text { Jarvinen et al., } 2009\end{array}$ \\
\hline
\end{tabular}


White Paper for the Planetary Science Decadal Survey of 2023-2033

\section{Conclusion:}

To advance the understanding of the coupled physical (radiative-dynamic) interactions and processes within the Venusian atmosphere "modern" observations are essential. Therefore, we strongly advocate that NASA consider one or more new missions to Venus that includes the necessary state-of-the-art atmospheric measurements, and a mechanism to fund simulation efforts supporting the interpretation and assessment of the observations. Notably, Venus' near proximity to the Earth promotes fast trips and frequent launch windows. Therefore, not only can data be quickly attained at low cost due to Venus' proximity to Earth, it can be quickly assessed using available simulation tools. As such, new and frequent missions to Venus as advocated in this white paper would produce timely and potentially paradigm-changing insights into how Venus' atmosphere works, as well as other terrestrial planet atmospheres within and beyond the Solar System.

\section{References}

Cover Image: Maciej Zborowski; Taylor et al. 2018; Kremic et al. 2018;

https://slideplayer.com/slide/6998149/

https://www.esa.int/Science_Exploration/Space_Science/Venus_Express/Where did Venus_s_water_go

https://www.nasa.gov/feature/jpl/the-return-to-venus-and-what-it-means-for-earth

http://akatsuki.isas.jaxa.jp/en/ https://www.northropgrumman.com/space/vamp/

Fukuhara, T., et al. (2017). Nature Geoscience. https://doi.org/10.1038/ngeo2873

Gérard, J. C., et al. (2017). Space Science Reviews. https://doi.org/10.1007/s11214-017-0422-0

Horinouchi, T., et al. (2020). Science. https://doi.org/10.1126/science.aaz4439

Imamura, T., et al. (2020). Space Science Reviews. https://doi.org/10.1007/s11214-020-00703-9

Jarvinen, R., et al. (2009). Annales Geophysicae. https://doi.org/10.5194/angeo-27-4333-2009

Kouyama, T., et al. (2017). Geophys. Res. Letters. https://doi.org/10.1002/2017GL075792

Ledvina, S. A., et al. (2016). AAS/Division for Planetary Sciences Meeting Abstracts \#48.

Ledvina, S. A., et al. (2008). Space Science Reviews. https://doi.org/10.1007/s11214-008-9384-6

Lundin, R., et al. (2011). Icarus. https://doi.org/10.1016/j.icarus.2011.06.034

Ma, Y. J., et al. (2013). J. Geophys. Res. Space Physics. https://doi.org/10.1029/2012JA018265

Morellina, S., et al. (2020). Icarus. https://doi.org/10.1016/j.icarus.2020.113761

Navarro, T., et al. (2018). Nature Geoscience. https://doi.org/10.1038/s41561-018-0157-x

Read, P. L., \& Lebonnois, S. (2018). Annual Review of Earth and Planetary Sciences. https://doi.org/10.1146/annurev-earth-082517-010137

Sánchez-Lavega, A., et al. (2017). Sp. Sci. Rev. https://doi.org/10.1007/s11214-017-0389-x

Schubert, G., et al. (2007). Geophysical Monograph Series. https://doi.org/10.1029/176GM07

Takagi, M., et al. (2018). J. Geophys. Res. Planets. https://doi.org/10.1002/2017JE005449

Taylor, F. W., et al. (2018). Space Science Reviews. https://doi.org/10.1007/s11214-018-0467-8

VEXAG19: Goals, Objective, and Investigations for Venus Exploration. https://www.lpi.usra.edu/vexag/reports/VEXAG_Venus_GOI_Current.pdf

VEXAG19_Tech: Venus Technology Plan.

https://www.lpi.usra.edu/vexag/reports/VEXAG_Venus_Techplan_Current.pdf 